\title{
Comparison of NICE Guideline CG149 and the Sepsis Risk Calculator for the Management of Early-Onset Sepsis on the Postnatal Ward
}

\author{
Jessica Alexandra Kimpton ${ }^{a}$ Amit Vermab $^{b}$ Devangi Thakkar ${ }^{c}$ Sophia Teoh ${ }^{d}$ \\ Aarti Verma $^{\mathrm{e}}$ Chinthika Piyasena ${ }^{f}$ Cheryl Battersbyg, ${ }^{\mathrm{h}}$ for the Neonatal \\ Trainee-Led Research and Improvement Projects (NeoTRIPs) \\ aEpsom and St Helier's University Hospitals NHS Trust, Carshalton, UK; ${ }^{b}$ Guy's and St Thomas' NHS Foundation \\ Trust, London, UK; ${ }^{C}$ Northwick Park Hospital, Harrow, London, UK; ${ }^{\mathrm{d} C r o y d o n}$ University Hospital, Croydon, UK; \\ eSouthend University Hospital NHS Foundation Trust, London, UK; 'Evelina London Children's Hospital,

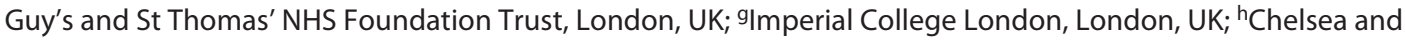 \\ Westminster Hospital, London, UK
}

\section{Keywords}

Neonate $\cdot$ Sepsis $\cdot$ Antibiotic $\cdot$ Postnatal ward $\cdot$ Infant

\begin{abstract}
Introduction: The NICE guideline CG149 has increased the number of well infants receiving antibiotics for suspected early-onset sepsis (EOS). The Kaiser Permanente sepsis risk calculator (SRC) has safely and dramatically reduced investigations and antibiotics for suspected EOS in the USA. This study evaluates the current management of suspected EOS against the NICE guideline CG149 and the SRC. Methods: This study is a prospective, multicentre, observational study across 13 neonatal units in London. Infants were born between June and August 2019 at $\geq 34$ weeks gestation and commenced on antibiotics for suspected EOS and cared for on postnatal/transitional care wards. Data were prospectively recorded: risk factors, clinical indicators, investigations, and results. Outcome measures included the following: (1)
\end{abstract}

karger@karger.com www.karger.com/neo

Karger $\stackrel{\text { ' }}{5}$

BOPEN ACCESS
(C) 2021 The Author(s)

Published by S. Karger AG, Basel

This is an Open Access article licensed under the Creative Commons Attribution-NonCommercial-4.0 International License (CC BY-NC) (http://www.karger.com/Services/OpenAccessLicense), applicable to the online version of the article only. Usage and distribution for commercial purposes requires written permission. incidence of EOS and (2) proportion of infants recommended for antibiotics by NICE versus theoretical application of SRC. Results: 1,066/8,856 (12\%) infants on postnatal/transitional care wards received antibiotics, 7 of whom had a positive blood culture (group B Streptococcus $=6$ and Escherichia coli = 1), making the EOS incidence $0.8 / 1,000$ infants. Six hundred one infants had data for SRC analysis, which recommended "antibiotics" or "blood culture" for 130/601 (21.6\%) infants using an EOS incidence of $0.5 / 1,000$ versus 527/601 (87.7\%) if NICE was applied. Conclusions: Currently, $12.0 \%$ of infants on postnatal/transitional care wards receive antibiotics for suspected EOS. The SRC could dramatically reduce antibiotic use, but further prospective studies are required to evaluate safety of SRC implementation.

(C) 2021 The Author(s)

Published by S. Karger AG, Basel

Jessica Alexandra Kimpton and Amit Verma are joint first authors to this work.
Correspondence to:

Jessica Alexandra Kimpton, jessica.kimpton@ doctors.org.uk

Cheryl Battersby, c.battersby@imperial.ac.uk 


\section{Introduction}

Early-onset sepsis (EOS) in neonates is defined as bacteraemia or bacterial meningitis within $72 \mathrm{~h}$ of birth. Despite improvements in antenatal care and provision of maternal intrapartum antibiotics, it remains an important cause of morbidity in neonates, particularly those born preterm [1]. In high-income settings, it occurs in $0.7 / 1,000$ live births ( $42 \%$ of whom were term infants) and is responsible for 5.6/1,000 neonatal admissions [2]. Early identification and management of EOS is vital to avoid adverse outcomes.

In 2012, the National Institute for Clinical Excellent (NICE) published the "Neonatal infection (early onset): antibiotics for prevention and treatment clinical guideline (CG149)" [3]. This guideline intended to provide clinicians in the UK with a consistent, safe framework for performing neonatal septic screens, promote antibiotic stewardship, reduce infant mortality, and reduce neonatal unit admissions and length of hospital stay. In reality, this guideline has increased investigations, antibiotic usage, duration of treatment, and hospital stay for many well newborns [4]. Investigations and treatments are associated with pain for the infant [5], antibiotic exposure (disrupting gut microbiota and contributing to antibiotic resistance) [6], separation of mother and baby, more formula milk supplementation [7], and greater costs to the National Health Service [4].

The Kaiser Permanente Research group developed an online sepsis risk calculator (SRC) to evaluate risk of neonatal EOS in infants $\geq 34$ weeks gestation [8]. The infant's EOS risk is calculated using the population's incidence of EOS and modified using objective information present at birth and the infant's evolving clinical presentation [9, 10]. This tool has been validated as a safe way to reduce the proportion of infants receiving empiric antibiotics without adverse effects $[11,12]$ and recommended by the Committee on the Fetus and Newborn of the American Academy of Pediatrics [13]. However, studies comparing theoretical effectiveness and safety of the SRC against NICE have produced conflicting results. One study reported that the SRC could reduce interventions and antibiotic usage [14], another that NICE guidelines identify asymptomatic cases earlier [15], and another raised concerns that the SRC may "miss" cases of EOS, particularly in the setting of chorioamnionitis [16].

One of the challenges of implementing the SRC in the $\mathrm{UK}$ is accurately defining the background incidence of EOS, which subsequently impacts clinical recommendations for the infant. The SRC offers a range of options between 0.1 and 4/1,000 live births and highlights 2 evidence-based options for an American population: 0.3/1,000 live births (Kaiser Permanente Northern California's incidence of EOS) and 0.5/1,000 live births (Centre for Disease Control and Prevention national incidence of EOS) [14]. However, the incidence of EOS varies geographically, not only by country but also between different regions [15].

\section{Aims}

1. To calculate the incidence of EOS in a population of infants born $\geq 34$ weeks gestation cared for on the postnatal/transitional care wards in London.

2. To compare the proportion of infants recommended antibiotics by the NICE guideline CG149 versus theoretical application of the SRC.

\section{Methods}

This prospective, multicentre, observational survey investigated management of EOS in London postnatal/transitional care units. All 26 London neonatal units were invited to take part via email. Local teams comprised a supervising consultant paediatrician and trainees in paediatrics.

Infants included those born $\geq 34$ weeks gestation between June 3, 2019, and August 2, 2019, commenced on antibiotics for suspected EOS and managed on the postnatal/transitional care ward. Infants admitted to the neonatal unit immediately after birth were excluded.

The study protocol and data collection forms were developed a priori [17]. Local teams obtained data from clinical records on background demographics, antenatal risk factors, clinical status, investigations, and antibiotic usage. Anonymized data were uploaded to a central database for analysis.

Risk estimates for the SRC were computed on Microsoft Excel using the Kaiser Permanente multivariate model equation [10]. We compared an EOS incidence of 0.5/1,000 infants to our EOS incidence of $0.8 / 1,000$ infants $\geq 34$ weeks cared for on the postnatal ward. The final risk estimate ("EOS risk after clinical exam") was obtained according to the SRC's clinical variables. Infants with other clinical signs were assigned "equivocal," and those admitted to the neonatal unit or with respiratory distress $>4 \mathrm{~h}$ after birth were assigned to "clinical illness." For infants with EOS, clinical recommendations were confirmed against the online SRC [8].

\section{Results}

Thirteen neonatal units in London contributed data. One thousand sixty-six of the $8,856(12.0 \%)$ infants $\geq 34$ weeks gestation born and managed on the postnatal/transitional care ward received antibiotics for suspected EOS during the study period (Table 1; Fig. 1). 
Table 1. Incidence of bacteraemia and bacterial meningitis across London neonatal units

\begin{tabular}{lr}
\hline EOS data & $N$ \\
\hline Infants $\geq 34$ weeks gestation cared for on the postnatal ward & 8,856 \\
Infants $\geq 34$ weeks gestation screened and treated for suspected EOS and cared for on the postnatal ward & 1,066 \\
Blood cultures available & 910 \\
Blood culture results & 19 \\
$\quad$ Positive blood cultures & 12 \\
Considered contaminants & 7 \\
Bacterial pathogen & 2 \\
CSF culture results & 2 \\
Positive CSF cultures & 0 \\
Considered contaminated/viral CSF cultures & 0.79 \\
Bacterial pathogen & 2 \\
EOS incidence/1,000 infants & \\
\hline EOS, early-onset sepsis; CSF, cerebrospinal fluid. & \\
\hline
\end{tabular}

Table 2. Comparing the proportion of infants managed for EOS by NICE versus the SRC

\begin{tabular}{lllrr}
\hline Clinical recommendation & Vitals & $\begin{array}{l}\text { NICE, } \\
\text { total (\%) }\end{array}$ & $\begin{array}{l}\text { SRC: EOS incidence } \\
0.5 / 1,000 \text { infants, total (\%) }\end{array}$ & $\begin{array}{l}\text { SRC: EOS incidence } \\
0.8 / 1,000 \text { infants, total (\%) }\end{array}$ \\
\hline No culture, no antibiotics & Routine vitals & $74(12.3)$ & $396(65.9)$ & $301(50.1)$ \\
No culture, no antibiotics & Vitals every 4 h for 24 h & & $75(12.5)$ & $121(20.1)$ \\
Blood culture & Vitals every 4 h for 24 h & & $75(12.5)$ & $97(16.1)$ \\
Empiric antibiotics & Vitals per NICU & $527(87.7)$ & $55(9.2)$ & $82(13.6)$ \\
Total & & $601(100)$ & $601(100)$ & $601(100)$ \\
\hline
\end{tabular}

EOS, early-onset sepsis; SRC, sepsis risk calculator.

\section{Incidence of EOS}

19/910 blood cultures had positive culture results, of which 7 were pathogens (Table 1; online suppl. Table 1; see www.karger.com/doi/10.1159/000518059 for all online suppl. material). Organisms included group B Streptococcus $=6$ and Escherichia coli $=1$. There were no cerebrospinal fluid results indicative of bacterial meningitis. The overall incidence of EOS in our study population was $0.8 / 1,000$ infants (95\% CI: 0.3/1,000-1.6/1,000).

\section{Theoretical Impact of SRC versus NICE}

Six hundred one infants had data available for the SRC versus NICE analysis. Two hundred seventy-four (46\%) were female, mean (range) gestation $39+4(34+0-42+0)$ weeks, birth weight mean (range) 3,366 g (1,830-5,025 g). 527/601 (87.7\%) met NICE guidelines for antibiotics. When the SRC was applied at an EOS incidence of $0.5 / 1,000$ infants, "empiric antibiotics" were recommended in 55/601 (9.2\%); "blood culture" in 75/601

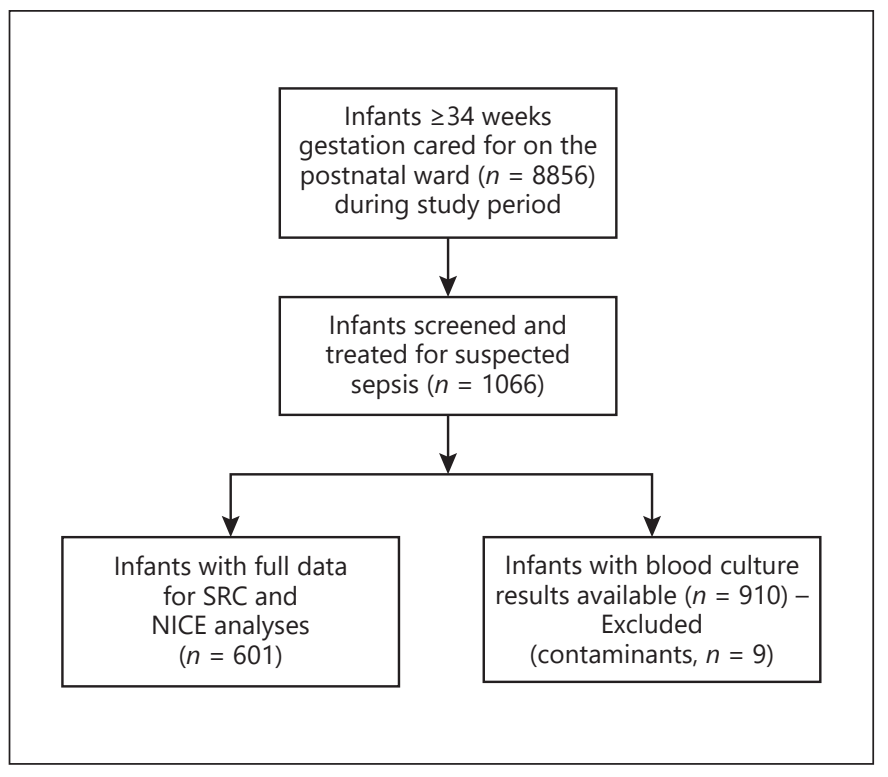

Fig. 1. Consort flow diagram. 


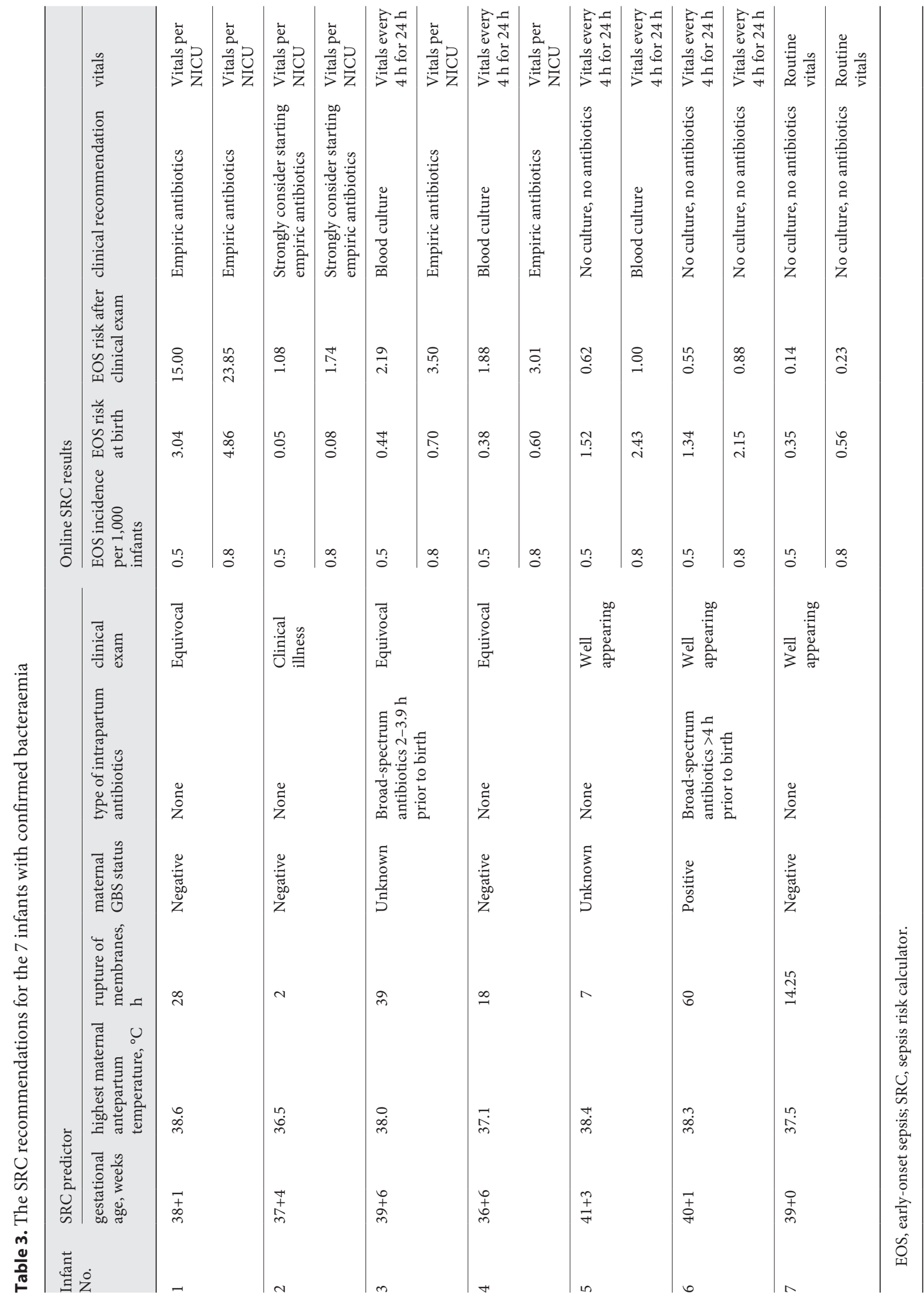


(12.5\%); "no culture, no antibiotics" but enhanced vitals in 75/601 (12.5\%); and no culture, no antibiotics, and routine vitals in 369/601 (65.9\%) (Table 2). Compared to the current practice influenced by NICE, theoretical application of the SRC at an EOS incidence of 0.5/1,000 infants could reduce antibiotics from 601 to 55 infants ( $90.8 \%$ reduction). If a more cautious approach was taken and antibiotics were administered to infants recommended for "blood culture" (75/601) in addition to "empiric antibiotics" (55/601), 130/601 (21.6\%) would be treated with antibiotics $(78.4 \%$ reduction compared to the current practice). Table 2 compares the SRC's advice at 0.5/1,000 infants to our EOS incidence of 0.8/1,000 infants.

\section{Confirmed EOS Cases}

Of the 7 infants with bacteraemia, the SRC recommended 2 infants for empiric antibiotics, 2 infants for blood culture with enhanced observations, and 2 infants for enhanced observations only (Table 3 ). NICE recommended 6 of the 7 infants for antibiotics. One infant did not meet either SRC or NICE criteria for antibiotics but received antibiotics due to a combination of clinical concerns. Adjusting the background incidence of EOS from 0.5 to $0.8 / 1,000$ infants escalated clinical advice for 3 infants.

\section{Discussion}

This multicentre, prospective study estimated the incidence of EOS and determined the proportion of infants recommended antibiotics by NICE in comparison with theoretical application of the SRC. Over a 2-month period, $12.0 \%$ of infants $\geq 34$ weeks gestation managed on postnatal/transitional care wards received antibiotics for suspected EOS, the majority (87.7\%) in line with NICE. Theoretical application of the SRC at an EOS incidence of 0.5/1,000 infants could reduce antibiotics from 601 (current practice influenced by NICE) to 55 infants (recommended "antibiotics" by the SRC) (90.8\% reduction). If a more cautious approach was taken and infants at intermediate risk (those recommended for a blood culture and a period of observation) were given antibiotics rather than observed, a 78.4\% reduction could still be achieved. Goel et al. [14] projected that the SRC could reduce antibiotics by $74 \%$ (relative reduction) compared with NICE. However, their study included the entire eligible birth cohort rather than just infants managed on the postnatal/ transitional care ward.
Under the NICE guideline CG149, empiric antibiotics are often commenced due to concerns of maternal sepsis, fever, or chorioamnionitis. Women often have pyrexia in labour, and given the maternal morbidity associated with chorioamnionitis, the subjective diagnosis of invasive infection, and the low thresholds for treatment, many women receive intrapartum antibiotics [18]. Intrapartum antibiotics have greatly reduced EOS [19], but newborns still receive antibiotics because intravenous antibiotic therapy for invasive maternal infection is a "red flag" for empiric antibiotics in the newborn under the NICE guidance CG149 [3]. The protective effect of maternal antibiotics on EOS was demonstrated in the multivariate SRC model [13], leading to reduced risk estimates and fewer antibiotics in well infants. In the new NICE 2021 guidance NG195 [20], parenteral antibiotics are no longer a risk factor, and chorioamnionitis and intrapartum fever are considered as 2 separate risk factors [21].

Our EOS incidence of 0.8/1,000 infants for Greater London approximated the neonIN infection surveillance network incidence of $0.7 / 1,000$ [2] and that of Morris et al. [15] (0.4-0.7/1,000 infants) for the other part of the UK. However, our EOS incidence is only an estimate, and the confidence intervals are wide due to the infrequency of EOS and our short observational period of 2 months. Changes to EOS incidence result in noticeable differences in the SRC's clinical recommendations, as demonstrated by our findings at an EOS incidence of 0.5/1,000 versus $0.8 / 1,000$ infants. Thus, we recommend regional surveillance studies to monitor the incidence of EOS. However, caution should be taken before adjusting the SRC to local incidence rates, as the model may require formal recalibration [22].

Missed cases of EOS are rare in resource-rich locations given its infrequency and the quality of neonatal care [11]. All 7 cases of EOS in our study received antibiotics, but 1 infant did not meet NICE or SRC criteria for antibiotics. This infant received antibiotics due to a combination of clinical concerns, highlighting the importance of using clinical intuition as well as guidelines, as not all infants have early clinical signs or typical maternal risk factors [15]. Evidence on missed cases is reported to be comparable between the SRC and conventional management [23]. However, studies evaluating the SRC in the UK are limited and often theoretical in design.

Randomized controlled trials evaluating the safety of the SRC may not be feasible because of the low incidence of EOS in high- and middle-income countries. Future studies should include concurrent data collection dur- 
ing implementation and after implementation of the SRC using routine electronic patient records and record linkage.

We are currently undertaking a prospective observational study comparing the number of "missed cases" of EOS using NICE CG149 versus SRC across neonatal units in Greater London [24]. We recommend neonatal units implement a similar local system to monitor for missed cases.

There are other considerations for neonatal units considering implementing the SRC. The SRC recommends some infants at intermediate risk to have a blood culture, enhanced observations, and initially withholding antibiotics, which is not a common practice in the UK. Health care providers should consider the available resources within their health care settings and whether enhanced monitoring and serial clinical examinations are feasible to identify evolving EOS cases. This decision should be balanced with the potentially large reduction in newborns requiring antibiotics at birth and associated reduction in workload [25]. In addition, if a blood culture is taken, aseptic techniques should be used to avoid contaminants and prompt processing and reporting systems available to prevent delays to antibiotic administration and cessation.

There are limitations to this study. (1) This study is limited to infants $\geq 34$ weeks gestation who received antibiotics and were cared for on the postnatal ward, and we did not obtain data for infants admitted to NICU or those who did not receive antibiotics. This limits our ability to determine compliance with NICE guidelines, as we do not have information on non-use of antibiotics. (2) The application of the SRC is theoretical and applied to a cohort of infants who were already identified as requiring antibiotics. (3) The incidence of EOS was based on the assumption that the infants who did not have a blood culture did not have sepsis. (4) There were missing data for the SRC comparison, which reduced the number of infants in this part of the analysis.

\section{Conclusion}

NICE guidance leads to a large proportion (12.0\%) of infants $\geq 34$ weeks on postnatal/transitional care wards to receive antibiotics for suspected EOS. The SRC, if used conservatively, could reduce antibiotic use by $78.4 \%$ using an EOS incidence of 0.5/1,000 infants. Prospective studies are required to evaluate the safety and the final clinical outcomes of the SRC.

An Observational Study on Neonatal Early-Onset Sepsis

\section{Acknowledgment}

NeoTRIPs Team: Apoorva Aiyengar, Narendra Aladangady, Upama Banerjee, Akif Barlas, Madeline Barnett, Tristan Bate, Andrea Beaton, Claire Caldwell, Andrew Chapman, Rose Cherian, Liyan Chow, James Cooper, Sarah Crew, Anne Davies, Akrum Elhussein, Jonathan Elliot, Malaz Elsaddig, Joy Eribo, Igor Fierens, Sorana Galu, Rebecca Gaunt, Sunit Godambe, Laila Hamidi, Chantal Hathaway, Eleanor Hulse, Karen Job, Soujyana Kalyanam, Geraint Lee, Joanna Mena, Joselyn Morris, Elizabeth Lek, Luke Mills, Alex Morton, Sam Nailor, Richard Nicholl, Christie Nobel, Catherine O'Sullivan, Siddharth Paliwal, Thushara Latha Perumal, Emily Prior, Heather Reid, Emma Schofield, Ana Serrano-Llop, Katherine Styles, Justinas Teiserskas, Jeanne Uhiriwe, Lucy Webster, Salim Yasin, Louis Yee, and Ka Yu Yung. The authors thank Professor Karen Puopolo and Kaiser Permanente Group for providing the SRC equation.

\section{Statement of Ethics}

The study was deemed service evaluation by the Chair and Approvals Officer of the London South East REC Committees and did not require ethical approval or patient consent.

\section{Conflict of Interest Statement}

Dr. Battersby reports grants and personal award funded by the National Institute for Health Research, personal fees from Chiesi Pharmaceuticals and AbbVie Pharmaceuticals, and is deputy chair of the NIHR Health Technology Assessment Prioritisation Committee for hospital-based care. Dr. Kimpton receives payment as a research editor for the $B M J$. The other authors do not have any conflicts of interest to declare.

\section{Funding Sources}

This research did not receive funding from any funding agency in the public, commercial, or not-for-profit sectors.

\section{Author Contributions}

All authors contributed to the study conception and design. Data were collected by the NeoTRIPs team. Data analysis and interpretation was performed by Jessica Kimpton, Amit Verma, and Devangi Thakkar. The first draft of the manuscript was written by Jessica Kimpton. All authors commented on previous versions of the manuscript and approved the final manuscript. 


\section{References}

1 Stoll BJ, Hansen NI, Sánchez PJ, Faix RG, Poindexter BB, Van Meurs KP, et al. Early onset neonatal sepsis: the burden of group B Streptococcal and E. coli disease continues. Pediatrics. 2011;127(5):817-26.

2 Cailes B, Kortsalioudaki C, Buttery J, Pattnayak S, Greenough A, Matthes J, et al. Epidemiology of UK neonatal infections: the neonIN infection surveillance network. Arch Dis Child Fetal Neonatal Ed. 2018;103(6):F547.

3 NICE. Neonatal infection (early onset): antibiotics for prevention and treatment CG149 2012. 2020. Available from: https://www.nice. org.uk/guidance/cg149.

4 Mukherjee A, Davidson L, Anguvaa L, Duffy DA, Kennea N. NICE neonatal early onset sepsis guidance: greater consistency, but more investigations, and greater length of stay. Arch Dis Child Fetal Neonatal Ed. 2015; 100(3):F248-9.

5 Williams MD, Lascelles BDX. Early neonatal pain-a review of clinical and experimental implications on painful conditions later in life. Front Pediatr. 2020;8:30.

6 Klingenberg C, Kornelisse RF, Buonocore G, Maier RF, Stocker M. Culture-negative earlyonset neonatal sepsis: at the crossroad between efficient sepsis care and antimicrobial stewardship. Front Pediatr. 2018;6:285.

7 Mukhopadhyay S, Lieberman ES, Puopolo KM, Riley LE, Johnson LC. Effect of early-onset sepsis evaluations on in-hospital breastfeeding practices among asymptomatic term neonates. Hosp Pediatr. 2015;5(4):203-10.

8 Northern California Kaiser-Permanente. Neonatal early-onset sepsis calculator. Available from: https://neonatalsepsiscalculator. kaiserpermanente.org/InfectionProbabilityCalculator.aspx.

9 Escobar GJ, Puopolo KM, Wi S, Turk BJ, Kuzniewicz MW, Walsh EM, et al. Stratification of risk of early-onset sepsis in newborns $\geq 34$ weeks' gestation. Pediatrics. 2014;133(1): $30-6$.
10 Puopolo KM, Draper D, Wi S, Newman TB, Zupancic J, Lieberman E, et al. Estimating the probability of neonatal early-onset infection on the basis of maternal risk factors. Pediatrics. 2011;128(5):e1155-63.

11 Kuzniewicz MW, Puopolo KM, Fischer A, Walsh EM, Li S, Newman TB, et al. A quantitative, risk-based approach to the management of neonatal early-onset sepsis. JAMA Pediatr. 2017;171(4):365-71.

12 Leonardi BM, Binder M, Griswold KJ, Yalcinkaya GF, Walsh MC. Utilization of a neonatal early-onset sepsis calculator to guide initial newborn management. Pediatr Qual Saf. 2019;4(5):e214

13 Puopolo KM, Benitz WE, Zaoutis TE. Management of neonates born at $\geq 35$ 0/7 weeks' gestation with suspected or proven early-onset bacterial sepsis. Pediatrics. 2018;142(6): e20182894.

14 Goel N, Shrestha S, Smith R, Mehta A, Ketty $M$, Muxworthy $H$, et al. Screening for early onset neonatal sepsis: NICE guidance-based practice versus projected application of the Kaiser permanente sepsis risk calculator in the UK population. Arch Dis Child Fetal Neonatal Ed. 2020;105(2):118.

15 Morris R, Jones S, Banerjee S, Collinson A, Hagan $\mathrm{H}$, Walsh $\mathrm{H}$, et al. Comparison of the management recommendations of the Kaiser permanente neonatal early-onset sepsis risk calculator (SRC) with NICE guideline CG149 in infants $\geq 34$ weeks' gestation who developed early-onset sepsis. Arch Dis Child Fetal Neonatal Ed. 2020 Nov;105(6):5816.

16 Pettinger KJ, Mayers K, McKechnie L, Phillips B. Sensitivity of the Kaiser permanente early-onset sepsis calculator: a systematic review and meta-analysis. EClinicalMedicine. 2020;19:100227.

17 Thakkar D, Verma A, Verma A, Teoh S, Piyasena C, Battersby C. Management of early onset sepsis in neonates 2019.2020. Available from: https://neotripsorg.files.wordpress. com/2019/11/early-onset-sepsis-study-protocol-final.pdf

18 Higgins RD, Saade G, Polin RA, Grobman WA, Buhimschi IA, Watterberg K, et al. Evaluation and management of women and newborns with a maternal diagnosis of chorioamnionitis: summary of a workshop. Obstet Gynecol. 2016;127(3):426-36.

19 Schrag SJ, Zywicki S, Farley MM, Reingold AL, Harrison LH, Lefkowitz LB, et al. Group $B$ streptococcal disease in the era of intrapartum antibiotic prophylaxis. N Engl J Med. 2000;342(1):15-20.

20 NICE. Neonatal infection: antibiotics for prevention and treatment NICE guideline [NG195]. Accessed from: https://www.nice. org.uk/guidance/NG195. Accessed May 2021.

21 NICE. Neonatal infection: antibiotics for prevention and treatment. Draft for consultation, December 2020. National Institute for Health and Care Excellence; 2020 [cited 2021. Available from: https://www.nice.org.uk/ guidance/GID-NG1011//documents/draftguideline.

22 Benitz WE, Achten NB. Technical assessment of the neonatal early-onset sepsis risk calculator. Lancet Infect Dis. 2021;21(5):e134-40.

23 Achten NB, Klingenberg C, Benitz WE, Stocker M, Schlapbach LJ, Giannoni E, et al. Association of use of the neonatal early-onset sepsis calculator with reduction in antibiotic therapy and safety: a systematic review and meta-analysis. JAMA Pediatr. 2019;173(11): 1032-40.

24 Neonatal Trainee-Led Research and Improvement Projects (NeoTRIPs). Pan-London early-onset sepsis observational study. 2020. Research protocol available via: https:// neotrips.org/. Accessed 2021 April.

25 Deshmukh M, Mehta S, Patole S. Sepsis calculator for neonatal early onset sepsis: a systematic review and meta-analysis. J Matern Fetal Neonatal Med. 2019;34:1832-40. 